Journal of Biological Systems, Vol. 15, No. 2 (2007) 169-184

(C) World Scientific Publishing Company

\title{
A SIMPLE SI MODEL WITH TWO AGE GROUPS AND ITS APPLICATION TO US HIV EPIDEMICS: TO TREAT OR NOT TO TREAT?
}

\author{
ROXANA LÓPEZ \\ Department of Mathematics \\ Universidad Nacional Mayor de San Marcos \\ Lima, Lima 1, Peru \\ rlopezc@unmsm.edu.pe \\ YANG KUANG* and ABDESSAMAD TRIDANE ${ }^{\dagger}$ \\ Department of Mathematics, Arizona State University \\ Tempe, AZ 85287, USA \\ *kuang@asu.edu \\ †tridane@math.la.asu.edu \\ Received 6 December 2006 \\ Revised 7 January 2007
}

\begin{abstract}
The objective of this paper is to study the global dynamics of a simple SI model with two explicit age groups and apply the findings to the HIV dynamics in the United States. Specifically, we would like to explore the long term HIV dynamics to answer questions such as what will happen to human population level if all the demographical and epidemiological parameters stay constant. We also wonder if treatment alone will actually slow the spread of HIV or not. We divide the population into juvenile and adult groups. Only adults are assumed to be sexually active and we implicitly assume that the sex ratio is constant. We also assume that infected adults may produce both susceptible newborns and infected newborns. The model is fit with parameters from the HIV epidemic in the US. It produces an optimistic outcome: if nothing changes, the USA infected population may be halved in about 20 years. However, if treatment is found to extend the life expectance of infected individuals to 30 years or more, then the number of infected adults may actually increase in the next 20 years or so. This creates a dilemma: to treat or not to treat?
\end{abstract}

Keywords: HIV Model; Basic Reproduction Number; Ratio Dependence; Global Stability.

\section{Introduction}

Throughout history, mankind has suffered many devastating infectious diseases and continues to be fearful of such diseases. In the 1918-1919 influenza pandemic, over 20 million people perished, including more than half a million Americans. Indeed,

\footnotetext{
* Corresponding author.
} 
many believe infectious diseases had greatly reduced the human population growth rates in the world prior to the 18 th century (Ref. 1, p. 302).

Disease as an important way to control the population is well known in animal population growth. Numerous experimental studies ${ }^{2}$ have shown that parasites can dramatically reduce ${ }^{3}$ host density and even drive host population to extinction. Specifically, it is widely regarded as the culprit of a number of high profile extinctions on various islands. ${ }^{4}$ This deterministic phenomenon is successfully modeled by a family of simple SI type host-parasite models. ${ }^{5-7}$ The most interesting properties of such models are their ability of generating host extinction dynamics with realistic parameter values and initial data. This is possible since such models naturally contain the proportion transmission term, which is frequently referred to as ratio-dependent functional response in the context of predator-prey models. ${ }^{8,9}$

Sexually transmitted diseases such as HIV epidemic exert extremely heavy burden in some populations, to say the least. ${ }^{10,11}$ Many developing countries are confronted with the devastating impact of the epidemics on the young and productive population groups. There is a growing number of children and adolescents suffering HIV in these countries, worsening the situation among the poor and marginalized groups. This inevitably results in the further diversion of resources from other health, welfare and educational priorities. This vicious cycle prompts the question such as how much worse this situation can become before it is over. Specifically one wonders how the population levels in such countries will be if all the demographical and epidemiological parameters stay constant and how to find more effective ways to reduce or stop the spread of these devastating epidemics. To this end, it is important to better understand the interaction of infectious diseases transmission process and population growth dynamics. This task is frequently carried out by formulating and studying plausible epidemiological models with vital dynamics in the form of differential equations. ${ }^{12}$

On the other hand, many people are puzzled by questions such as why some diseases develop suddenly and why some people can escape them? The simple and classical Kermack-McKendrick model $(1927)^{13}$ provided a plausible answer to these intriguing questions. Specifically, the Kermack-McKendrick model is an SIR model proposed to explain the rapid rise and fall in the number of infected patients observed in epidemics such as plague (London 1665-1666, Bombay 1906) and cholera (London 1865). It assumes that the population size is fixed (i.e. no births, deaths due to disease, or deaths by natural causes, which are unrealistic), incubation period of the infectious agent is instantaneous (again unrealistic), and duration of infectivity is the same as length of the disease. It also assumes a completely homogeneous population with no age, spatial, or social structure. In other words, it is a bare bone model with many directions for improvement. After more than half a century, the Kermack-McKendrick model was brought back to full life by Anderson and May. ${ }^{14}$ More complicated versions of the Kermack-McKendrick model that better reflect the actual biology and dynamics of a given disease are often used. 
Our main objective here is to study the global dynamics of a simple SI model with two explicit age groups and apply the findings to the HIV dynamics in the US. Specifically, we would like to explore the long term HIV dynamics to answer questions such as what will happen to human population level if all the demographical and epidemiological parameters stay constant; if necessary, what can be done to slow the spread of HIV before effective cures or vaccines are found; what are the effects of treatments.

We divide the population into juvenile and adult groups. Only adults are assumed to be sexually active and we implicitly assume that the sex ratio is constant. We also assume that infected adults may produce both susceptible newborns and infected newborns. In many developing countries, as many as $25 \%$ of the newborns from infected mothers are infected. We show that such models can produce the often observed and feared scenario of susceptible extinction. This suggests that age structure alone will not remove the total extinction dynamics in a typical ratiodependent predator-prey model. This model is fit with parameters from the HIV epidemic in the US. It produces an optimistic outcome: if nothing changes, the US infected population may be halved in about 20 years. However, if treatment is found to extend the life expectance of infected individuals to 30 years or more, then the number of infected adults may actually increase in the next 20 years or so. This creates a dilemma: to treat or not to treat?

\section{An SI Model with Two Age Groups}

Our model deals with a closed population of heterosexual individuals. The population is divided into two groups: the juvenile $(J)$ group (0-14 years) and adult $(A)$ group (15 years or older). The epidemics divides the population into two classes: susceptible class (1) and infected class (2). The juvenile and adult groups are each further divided into two classes. In the following, we define $J_{1}$ as the size of the susceptible juvenile class, $J_{2}$ as the size of the infected juvenile class, $A_{1}$ as the size of the susceptible adult class, and $A_{2}$ as the size of the infected adult class. We assume that infected individuals do not recover (such as HIV), juveniles are not sexually active and only adults can reproduce. Our model takes the following form.

$$
\left\{\begin{array}{l}
J_{1}^{\prime}=\beta_{1} A_{1}+(1-\xi) \beta_{2} A_{2}-\eta_{1} J_{1}-\mu J_{1}-m J_{1} N \\
A_{1}^{\prime}=\eta_{1} J_{1}-\frac{v A_{1} A_{2}}{A}-\alpha A_{1}-m A_{1} N \\
J_{2}^{\prime}=\xi \beta_{2} A_{2}-\eta_{2} J_{2}-\mu J_{2}-\gamma J_{2}-m J_{2} N \\
A_{2}^{\prime}=\eta_{2} J_{2}+\frac{v A_{1} A_{2}}{A}-\alpha A_{2}-\gamma A_{2}-m A_{2} N
\end{array}\right.
$$

where $A=A(t)=A_{1}(t)+A_{2}(t)$ and $N=N(t)=J_{1}(t)+J_{2}(t)+A_{1}(t)+$ $A_{2}(t)$. Notice that $f\left(A_{1}, A_{2}\right) \equiv A_{1} A_{2} /\left(A_{1}+A_{2}\right) \leq \min \left\{A_{1}, A_{2}\right\}$, we see that if we define $f(0,0)=0$, then it is continuous at $\left(A_{1}, A_{2}\right)=(0,0)$. In fact, $f\left(A_{1}, A_{2}\right)$ is Lipschitzian for $A_{1} \geq 0$ and $A_{2} \geq 0$. However, $f\left(A_{1}, A_{2}\right)$ is not differentiable at $\left(A_{1}, A_{2}\right)=(0,0)$. 
Most of the terms in system (2.1) are standard. The exception is the additional mortality term due to crowdedness (intraspecific competition) in each classes. Crowdedness or intraspecific competition occurs when individuals of the same population negatively affect each other's fitness by reducing the availability of one or more limiting resources. At the individual level, competition for resources can affect development, fertility and survival. At the population level, intraspecific competition for resources can give rise to logistic population growth. It is widely known that intraspecific competition enables the co-existence of competing species.

With the help of the model flow chart (Fig. 1), the meanings of almost all the terms are self-evident. Nevertheless, we briefly describe each and every term here. The susceptible juveniles are recruited from the births due to susceptible adults $\left(\beta_{1} A_{1}\right)$ and due to infected adults $\left((1-\xi) \beta_{2} A_{2}\right)$, reduced by maturing juveniles $\left(\eta_{1} J_{1}\right)$, baseline death process $\left(\mu J_{1}\right)$ and additional mortality due to crowdedness $\left(m J_{1} N\right)$. The susceptible adults are recruited from the maturing susceptible juveniles $\left(\eta_{1} J_{1}\right)$ and reduced by the infection $\left(v A_{1} A_{2} / A\right)$, the baseline death process $\left(\alpha A_{1}\right)$, and additional mortality due to crowdedness $\left(m A_{1} N\right)$. The infected juveniles are recruited from the births due to infected adults $\left(\left(\xi \beta_{2} A_{2}\right)\right.$, reduced by maturing juveniles $\left(\eta_{2} J_{2}\right)$, baseline death process $\left(\mu J_{2}\right)$ and additional mortality due to the disease $\left(\gamma J_{2}\right)$ and crowdedness $\left(m J_{1} N\right)$. The infected adults are recruited from the maturing infected juveniles $\left(\eta_{2} J_{2}\right)$ and newly infected adults $\left(v A_{1} A_{2} / A\right)$, reduced by the baseline death process $\left(\alpha A_{2}\right)$, and additional mortality due to the disease $\left(\gamma A_{2}\right)$ and crowdedness $\left(m A_{2} N\right)$.

We assume that the per capita birth rates $\beta_{j}, j=1,2$, maturation rates $\eta_{j}, j=$ 1,2 , baseline juvenile death rates $\mu$, adult baseline death rate $\alpha$, diseases-induced

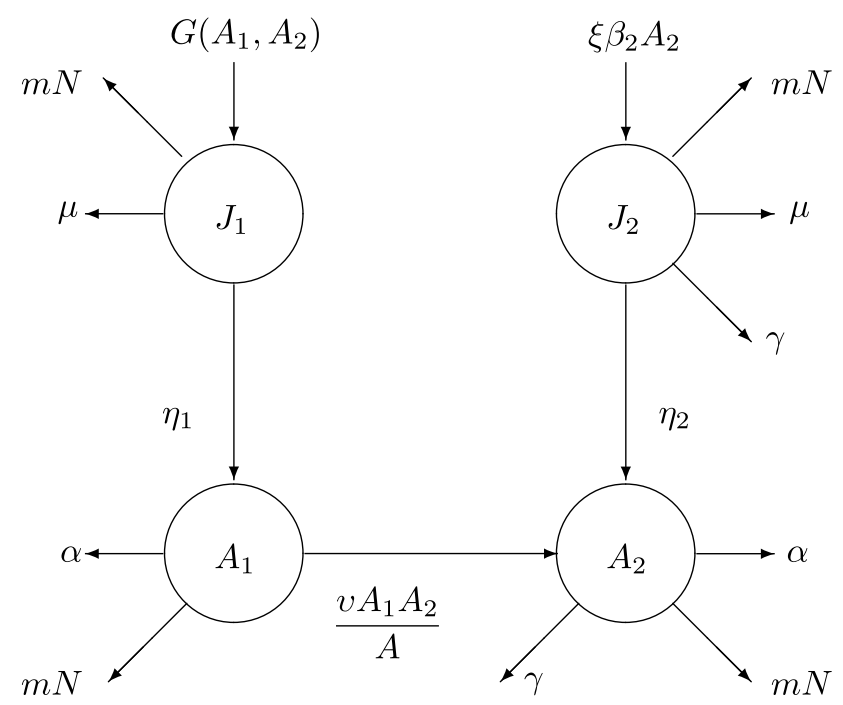

Fig. 1. Flow diagram for the structured SI model with two ages. Consider $G\left(A_{1}, A_{2}\right)=\beta_{1} A_{1}+$ $(1-\xi) \beta_{2} A_{2}$. 
death rate $\gamma$ and the crowdedness coefficient $m$ are positive. We assume that a fraction $\xi(0 \leq \xi \leq 1)$ of birth from infected adults are infected. We would like to mention here that this fraction is highly controllable, especially in the US. Due to biological reality, we also assume that $J_{i}(0)>0, A_{i}(0)>0, i=1,2$.

\section{Preliminary Analysis}

In this section, we establish some basic results for the solutions of the system (2.1) which will be needed in the subsequent work. In the following, $\mathbb{R}_{+}^{n}$ is defined as the set of positive vectors $x=\left(x_{1}, x_{2}, \ldots, x_{n}\right)$ with $x_{j}>0$ for $j=1,2, \ldots, n$. It is easy to see the functions on the right hand sides of the equations in system (2.1) are Lipschitzian which ensures the existence and uniqueness of the solutions. Due to the presence of the intraspecific competition in every classes, it is easy to show that solutions of the system (2.1) are eventually uniformly bounded.

The key value governing the time evolution of these equations is the so-called reproduction number (ratio) $R_{0}$. It is defined as the number of secondary infections caused by a single primary infection. It determines the number of people infected by contact with a single infected person before his death or recovery. This number is also referred in literature as basic reproduction (reproductive) ratio, or basic reproduction (reproductive) number. When $R_{0}<1$, each infected individual will infect fewer than one person before dying or recovering, so the disease will die out. When $R_{0}>1$, each infected individual will infect more than one person, so the disease will spread. $R_{0}$ is probably the single most important quantity in epidemiology research and applications. For our model, we can see that

$$
R_{0}=\left(\frac{\xi \beta_{2}}{\alpha+\gamma}\right)\left(\frac{\eta_{2}}{\eta_{2}+\mu+\gamma}\right) .
$$

This value is the average number of new infected juveniles produced by one typical infected adult during its adulthood times the probability of surviving the infected juvenile stage. This is the same as the number of secondary infections caused by a single primary infection.

The following biologically meaningful threshold value is also useful in our mathematical presentation.

$$
R_{1}=\left(\frac{\beta_{1}}{\alpha}\right)\left(\frac{\eta_{1}}{\eta_{1}+\mu}\right), \quad R_{2}=\frac{v}{\gamma+\alpha} .
$$

Biologically, $R_{1}$ is the average amount of new susceptible juveniles produced by one susceptible adult during its adulthood times the probability of surviving the susceptible juvenile stage. $R_{2}$ is the average number of contacts per infective adult per day times average period of infectivity. $R_{1}$ and $R_{0}$ are called reproduction ratios of the susceptible and infected classes, respectively. $R_{2}$ is called the infectious contact number. 
The system (2.1) has the trivial equilibrium $(0,0,0,0)$. Due to the fact that $f\left(A_{1}, A_{2}\right)$ is not differentiable at $\left(A_{1}, A_{2}\right)=(0,0)$, we cannot perform local stability analysis at this equilibrium by the standard linearization method.

We can establish the following result on the existence of the disease-free equilibrium.

Proposition 3.1. For system (2.1), if $R_{1}>1$, then the system has a unique disease-free equilibrium given by the expression

$$
J_{1}^{*}=\frac{\left(\alpha+m N_{1}^{*}\right) N_{1}^{*}}{\alpha+\eta_{1}+m N_{1}^{*}}, \quad A_{1}^{*}=\frac{\eta_{1} N_{1}^{*}}{\alpha+\eta_{1}+m N_{1}^{*}},
$$

where

$$
N_{1}^{*} \equiv N_{1} \equiv \frac{-\left(\eta_{1}+\mu+\alpha\right)+\sqrt{\left(\eta_{1}+\mu+\alpha\right)^{2}-4 \alpha\left(\eta_{1}+\mu\right)\left(1-R_{1}\right)}}{2 m} .
$$

For a susceptible extinction equilibrium to exist, we must have $\xi=1$. We have the following result.

Proposition 3.2. For system (2.1), if $R_{0}>1$ and $\xi=1$, then the system has a unique susceptible extinction equilibrium given by the expression

$$
J_{2}^{*}=\frac{\left(\alpha+\gamma+m N_{2}^{*}\right) N_{2}^{*}}{\alpha+\eta_{2}+\gamma+m N_{2}^{*}}, \quad A_{2}^{*}=\frac{\eta_{2} N_{2}^{*}}{\alpha+\eta_{2}+\gamma+m N_{2}^{*}},
$$

where

$$
N_{2}^{*} \equiv N_{2} \equiv \frac{-\left(\eta_{2}+\mu+2 \gamma+\alpha\right)+\sqrt{\Delta}}{2 m}
$$

with

$$
\Delta=\left(\eta_{1}+\mu+2 \gamma+\alpha\right)^{2}-4(\alpha+\gamma)\left(\eta_{2}+\mu+\gamma\right)\left(1-R_{2}\right)
$$

\section{Local and Global Stability Results}

In this section, we present both local and global stability results for the non-trivial equilibria of the system (2.1). All our conditions are in terms of the biologically meaningful threshold values defined in the previous section.

We present first the two local stability results. These results can be obtained by standard eigenvalue analysis. The proofs of these two results are straightforward since the Jacobian matrices in these two cases have a triangle block structure with a two by two block size. Therefore, we will just outline their proofs in the appendices.

The first one is for the disease-free equilibrium. Biologically, it states that if the susceptible class reproduction ratio is greater than one and the sum of the reproduction ratio for the infected class and its infectious contact number is no 
bigger than one, then the disease will be unable to invade a healthy population with small infected individuals.

Theorem 4.1. If $R_{1}>1$ and $R_{0}+R_{2} \leq 1$, then the disease-free equilibrium $\left(J_{1}^{*}, A_{1}^{*}, 0,0\right)$ is locally asymptotically stable in $\mathbb{R}_{+}^{4}$ for the system (2.1).

On the other hand, if the susceptible class reproduction ratio is no greater than one and the reproduction ratio for the infected class is bigger than one, then the disease can invade and persist in a healthy population with small infected individuals. This is basically what stated in the next local stability result that addresses the susceptible extinction equilibrium.

Theorem 4.2. If $R_{1} \leq 1, R_{0}>1$ and $\xi=1$, then the susceptible extinction equilibrium $\left(0,0, J_{2}^{*}, A_{2}^{*}\right)$ is locally asymptotically stable in $\mathbb{R}_{+}^{4}$ for the system (2.1).

We now present global stability results for the equilibria of the system (2.1). The biological implications of these global stability results will be addressed in the discussion section. Our first global stability result provides conditions for the disease to die out.

Theorem 4.3. If $R_{1}>\left(\eta_{1}+\mu\right)(v+\alpha) /\left(\eta_{1} \alpha\right)$ and $R_{2} \leq 1-R_{0}$, then $\lim \sup _{t \rightarrow \infty} N(t)>0$ and the disease-free equilibrium $\left(J_{1}^{*}, A_{1}^{*}, 0,0\right)$ is a global attractor in $\mathbb{R}_{+}^{4}$.

The conditions for the case when infected subpopulation eventually win the competition with the susceptible subpopulation eventually disappearing are given by the following theorem.

Theorem 4.4. If $\xi=1, R_{1} \leq 1$ and $R_{0}>\frac{\mu+\eta_{2}+\gamma}{\eta_{2}}$, then $\lim \sup _{t \rightarrow \infty} N(t)>0$ and the susceptible extinction equilibrium $\left(0,0, J_{2}^{*}, A_{2}^{*}\right)$ is a global attractor in $\mathbb{R}_{+}^{4}$.

To end this section, we present a very natural result that describes the devastating scenario of the extinction of the total population.

Theorem 4.5. If $\xi=1, R_{1} \leq 1$ and $R_{0} \leq 1$, then all solutions of the system (2.1) satisfies $\lim _{t \rightarrow \infty}\left(J_{1}(t), A_{1}(t), J_{2}(t), A_{2}(t)\right)=(0,0,0,0)$.

\section{Application to the US HIV Epidemics}

In the United States about 40,000 new HIV infections occur each year. More than $30 \%$ of these infections occur in women, and $60 \%$ occur in ethnic minorities. As of 2002 about 886,000 US residents were living with HIV/AIDS, and about 500,000 people had died of the disease since the epidemic began. 
Table 1. Estimated parameter values used in our simulation work for the US HIV epidemics.

\begin{tabular}{clc}
\hline Parameter & \multicolumn{1}{c}{ Meaning } & Value \\
\hline$\beta_{1}$ & $\begin{array}{l}\text { Per capita birth rate (SBR) } \\
\text { of an average susceptible adult }\end{array}$ & $0.02714\left(\mathrm{yr}^{-1}\right)$ \\
$\beta_{2}$ & $\begin{array}{l}\text { Per capita birth rate } \\
\text { of an average infected adult }\end{array}$ & $0.0015151851\left(\mathrm{yr}^{-1}\right)$ \\
$\mu$ & $\begin{array}{l}\text { Per capita juvenile death rate } \\
\text { (mean of JDR from 1992-2001) }\end{array}$ & $0.0038\left(\mathrm{yr}^{-1}\right)$ \\
$\alpha$ & $\begin{array}{l}\text { Per capita death rate } \\
\text { (mean of CDR from 2001-2004) }\end{array}$ & $0.0128683567\left(\mathrm{yr}^{-1}\right)$ \\
$\gamma$ & $\begin{array}{l}\text { Per capita HIV-related death rate } \\
\text { (reciprocal of mean infection period) }\end{array}$ & $0.0563115789\left(\mathrm{yr}^{-1}\right)$ \\
$v$ & $\begin{array}{l}\text { Per capita contact rate } \\
\text { (contact rate 2002) }\end{array}$ & $0.0451\left(\mathrm{yr}^{-1}\right)$ \\
$\eta_{1}, \eta_{2}$ & Maturation rates & $0.067\left(\mathrm{yr}^{-1}\right)$ \\
\hline
\end{tabular}

The average per capita birth rates of the susceptible and infected adults are calculated by the following formulas

$$
\begin{aligned}
& \beta_{1}=S B R=\frac{\text { total susceptible women pop. } \times \text { total fertility rate }}{\text { total women pop. }}\left(\mathrm{yr}^{-1}\right), \\
& \beta_{2}=I B R=\frac{\text { total infected women pop. } \times \text { total fertility rate }}{\text { total women pop. }}\left(\mathrm{yr}^{-1}\right)
\end{aligned}
$$

We assume that the natural baseline death rate is equal to the inverse of the average life expectancy at birth $(A L E)$

$$
\alpha=\frac{1}{A L E}\left(\mathrm{yr}^{-1}\right) .
$$

We assume that the juvenile death rate $(J D R)$ is the mean of juvenile mortality rates of two different years

$$
\mu=\frac{J D R(92 / 96)+J D R(97 / 01)}{2}\left(\mathrm{yr}^{-1}\right) .
$$

Once an individual is infected with HIV, the mean duration of infection ranges from 8.6-19 years. ${ }^{10,15}$ We assume that the average remaining life of an infected adult is equal to the inverse of the Mean Infection Period (MIP). Hence

$$
\gamma=\frac{1}{M I P}, \quad\left(\mathrm{yr}^{-1}\right)
$$

The Contact Rate is calculated by the formula

$$
v=\frac{\text { total new infected adult }(\text { in } 1 \text { year }) \times \text { total pop. }}{\text { total infected pop. } \times \text { total susceptible pop. }} .
$$



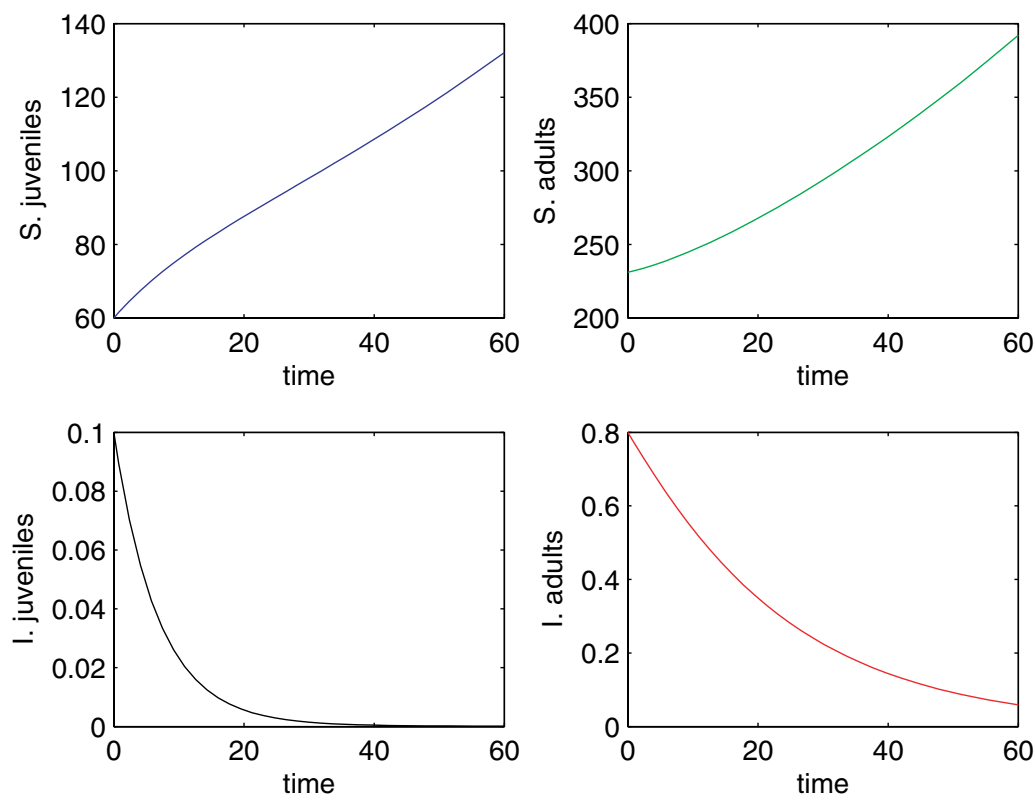

Fig. 2. Time unit is year. This figure depicts the simulation result for the US population data. The population unit is million. Parameter values are: $\beta_{1}=.02714 ; \mu_{1}=.0038 ; v=.0451 ; \alpha=.0129$; $\beta_{2}=.0015 ; \gamma=0.077 ; \mu_{2}=\mu_{1}+\gamma ; K=543119 ; m=\left(\beta_{1}-\alpha\right) / K ; \eta_{1}=0.067 ; \eta_{2}=0.067$; $\mu=.0038, \xi=0.1$. Initial population values are that of $2003: J_{1}(0)=60 \times 10^{6}, A_{1}(0)=231 \times 10^{6}$, $J_{2}(0)=0.1 \times 10^{6}, A_{2}(0)=0.8 \times 10^{6}$.

Since the total susceptible population is close to the total population, we see that

$$
v \approx \frac{\text { total new infected adult (in } 1 \text { year) }}{\text { total infected pop. }}
$$

Using these formulas, we obtain the following values of the parameters for the US HIV epidemics.

$$
\begin{aligned}
& \beta_{1}=14.14 / 1000=0.01414\left(\mathrm{yr}^{-1}\right), \\
& \beta_{2}=\frac{(87940)(2.08)}{120721354}=0.0015151851\left(\mathrm{yr}^{-1}\right), \\
& \mu=\frac{J D R(92-96)+J D R(97-01)}{2}=\frac{.0033+.0043}{2}=0.0038\left(\mathrm{yr}^{-1}\right), \\
& \alpha=1 / 77.71=0.0128683567\left(\mathrm{yr}^{-1}\right), \\
& \gamma=1 / 19=0.056315789\left(\mathrm{yr}^{-1}\right), \\
& v=40000 / 886000=0.0451\left(\mathrm{yr}^{-1}\right), \\
& \eta_{1}=\eta_{2}=1 / 15=0.067\left(\mathrm{yr}^{-1}\right) .
\end{aligned}
$$

We summarize these in Table 1. 
The carrying capacity of US due to the intraspecific competition is estimated as (Roxana 2006)

$$
K=543 \text { millions. }
$$

The parameter $m$ describing the intraspecific competition is calculated by the formula

$$
m=\frac{\beta_{1}-\alpha}{K}=2.34 \times 10^{-12}
$$

The US threshold values are

$$
\mathbf{R}_{1}=1.9956, \quad \mathbf{R}_{0}=7.6418 \times 10^{-4}, \quad \mathbf{R}_{2}=0.5018 .
$$

These threshold values satisfy Theorem 4.1 (but not Theorem 5.1) which implies that the system (2.1) has a locally asymptotically stable disease-free equilibrium.

\section{Discussion}

Due to the simplicity of our SI model with two age groups, we are able to carry out a systematical local and global stability analysis of it. An important feature of our work is that our analytical results are all presented in terms of the biologically meaningful threshold values. To facilitate a more detailed presentation of the biological implications of our mathematical findings, we summarize our main results for system (2.1) in Table 2 .

The local stability result Theorem 4.1 for the disease-free equilibrium suggests that in a disease without recovery, if the susceptible reproduction ratio is larger than 1 , then a unique disease-free equilibrium is feasible. If in addition, the sum of the infectious contact number and the infective reproduction ratio is less than one and the susceptible reproduction ratio is greater than the inverse of the product of the probability of surviving from the susceptible juvenile stage and the probability of dying during the susceptible adult stage, then our global stability result Theorem 4.3 states that the infected classes will eventually disappear and the susceptible classes approach a positive constant value.

Table 2. Local and global stability results of system (2.1). E stands for equilibrium, LAS stands for locally asymptotically stable and GS stands for globally stable.

\begin{tabular}{ll}
\hline Conditions & \multicolumn{1}{c}{ Results } \\
\hline (a) $R_{1}>1, R_{0}<1$ and $0<R_{2} \leq 1-R_{0}$ & The disease free E is LAS \\
(b) $R_{1}<1, R_{0}>1$ and $\xi=1$ & The susceptible extinction E is LAS \\
(c) $R_{1}>\frac{\left(\eta_{1}+\mu\right)(C+\alpha)}{\eta_{1} \alpha}$ and $R_{2} \leq 1-R_{0}$ & The disease free E is GS \\
(d) $\xi=1, R_{1} \leq 1$ and $R_{0}>\frac{\mu+\eta_{2}+\gamma}{\eta_{2}}$ & The susceptible extinction E is GS \\
(e) $\xi=1, R_{1} \leq 1$ and $R_{0} \leq 1$ & The total extinction E is GS \\
\hline
\end{tabular}


In a disease without recovery with any initial population size, if the infected reproduction ratio is greater than one, then a unique susceptible extinction equilibrium is feasible. Moreover, if the susceptible reproduction ratio is less than one, $100 \%$ of the babies from infected pregnant women are infected and the infected reproduction ratio is greater than the inverse of the probability to survive the infected juvenile stage, then our global stability result Theorem 4.4 states that the susceptible classes will eventually disappear and the infected classes approach a positive constant value.

Theorem 4.5 states that in a disease without recovery, if the susceptible and infected reproduction ratio is less than or equal to one, then the total population eventually disappear. This is intuitive as it simply states that if both the susceptible and infected cannot generate enough growth to replacing themselves, then they are doomed.

The most interesting findings are found in the application of our model to the US HIV epidemics. We found out through our model and our parameter estimation effort that the HIV infection will die out naturally with the HIV infected adults halved in less than 20 years if all the infection and demographic parameters stay where they are. Paradoxically, shall a treatment become available such that extending the life expectancy for HIV infected individuals from the current mean value of 13 years to 30 years or more, then the total HIV infected adults are expected to increase in the next 20 years. This, if true, certainly creates a dilemma to our society: to treat or not to treat? Sensible answers to this question must include factors that are excluded from our simple model.

\section{Acknowledgments}

The authors would like to thank Roger Jean, Hal Smith and Horst Thieme for their many valuable suggestions. This research is supported in part by DMS-0436341 and DMS/NIGMS-0342388. Correspondence should be directed to Yang Kuang.

\section{References}

1. Brauer F, Castillo-Chavez C, Mathematical Models in Population Biology and Epidemiology, Springer Verlag, 2001.

2. Ebert D, Lipsitch M, Mangin KL, The effect of parasites on host population density and extinction: experimental epidemiology with Daphnia and six microparasites, Am Nat 156:459-477, 2000.

3. Anderson RM, Theoretical basis for the use of pathogens as biological control agents of pest species, Parasitology 84:3-33, 1982.

4. McCallum H, Dobson A, Detecting disease and parasite threats to endangered species and ecosystems, Trends Ecol Evol 10:190-194, 1995.

5. Hwang T-W, Kuang Y, Deterministic extinction effect of parasites on host populations, J Math Biol 46:17-30, 2003.

6. Hwang T-W, Kuang Y, Host extinction dynamics in a simple parasite-host interaction model, Math Biosci Eng 2:743-751, 2005. 
7. Berezovsky F, Karev G, Song B, Castillo-Chavez C, A simple epidemic model with surprising dynamics, Math Biosci Eng 1:1-20, 2004.

8. Kuang Y, Beretta E, Global qualitative analysis of a ratio-dependent predator-prey system, J Math Biol 36:389-406, 1998.

9. Hsu S-B, Hwang T-W, Kuang Y, Global analysis of the Michaelis-Menten type ratiodependent predator-prey system, J Math Biol 42:489-506, 2001.

10. Kribs-Zaleta CM, Lee M, Román C, Wiley S, Hernández-Suárez CM, The effect of the HIV/AIDS epidemic on Africa's truck drivers, Math Biosci Eng 2:771-788, 2005.

11. Lopez R, Structured SI epidemic models with applications to HIV epidemic, PhD Thesis, Arizona State University.

12. Thieme H, Mathematics in Population Biology, Princeton University Press, Princeton, 2003.

13. Kermack W, McKendrick A, A contribution to mathematical theory of epidemics, Proc R Soc Lond A 115:700-721, 1927.

14. Anderson RM, May RM, Population biology of infectious diseases: part I, Nature 280:361-367, 1979.

15. Hyman JM, Li J, Stanley EA, The differential infectivity and staged progression models for the transmission of HIV, Math Biosci 155:77-109, 1999.

\section{Appendix A: Proof of Theorem 4.1}

From Proposition 3.1, we see that the condition $R_{1}>1$ ensures the uniqueness of the disease-free equilibrium. The Jacobian matrix of the system (2.1) evaluated at the disease-free equilibrium is of the form

$$
\mathbf{B}=\left(\begin{array}{cc}
\mathbf{B}_{1} & \mathbf{B}_{3} \\
\mathbf{0} & \mathbf{B}_{2}
\end{array}\right)
$$

where $\mathbf{B}_{1}, \mathbf{B}_{2}, \mathbf{B}_{3}, \mathbf{0}$ are $2 \times 2$ matrices and

$$
\begin{aligned}
& \mathbf{B}_{1}=\left(\begin{array}{cc}
-\left(\eta_{1}+\mu+m\left(J_{1}^{*}+N_{1}\right)\right) & \beta_{1}-m J_{1}^{*} \\
\eta_{1}-m A_{1}^{*} & -\left(\alpha+m\left(A_{1}^{*}+N_{1}\right)\right)
\end{array}\right), \\
& \mathbf{B}_{2}=\left(\begin{array}{cc}
-\left(\eta_{2}+\mu+\gamma+m N_{1}\right) & \xi \beta_{2} \\
\eta_{2} & v-\left(\alpha+\gamma+m N_{1}\right)
\end{array}\right), \\
& \mathbf{B}_{3}=\left(\begin{array}{cc}
-m J_{1}^{*} & (1-\xi) \beta_{2}-m J_{1}^{*} \\
-m A_{1}^{*} & -\left(v+m A_{1}^{*}\right)
\end{array}\right) .
\end{aligned}
$$

Clearly $\operatorname{tr}\left(\mathbf{B}_{1}\right)<0$. From the $J_{1}$ and $A_{1}$ equations, we see that $\beta_{1} \eta_{1}=(\alpha+$ $\left.m N_{1}\right)\left(\eta_{1}+\mu+m N_{1}\right)$. Hence, we have

$$
\operatorname{det}\left(\mathbf{B}_{1}\right)=m A_{1}^{*}\left(\eta_{1}+\mu+\beta_{1}+m N_{1}\right)+m J_{1}^{*}\left(\eta_{1}+\alpha+m N_{1}\right)>0 .
$$

In consequence, $\mathbf{B}_{1}$ has negative eigenvalues. Observe the assumption that $R_{2}<1$ implies that $\gamma+\alpha>v$. We have $\operatorname{tr}\left(\mathbf{B}_{2}\right)<0$ and

$$
\begin{aligned}
\operatorname{det}\left(\mathbf{B}_{2}\right)= & \left(\gamma+\eta_{2}+\mu\right)(\gamma+\alpha)\left(1-\left(R_{0}+R_{2}\right)\right)+\left(m N_{1}\right)^{2} \\
& +m N_{1}\left((\gamma+\alpha-v)+\eta_{2}+\mu+\gamma\right)>0,
\end{aligned}
$$

since $R_{2}+R_{0} \leq 1$. In consequence, $\mathbf{B}_{2}$ has negative eigenvalues. Therefore, the disease-free equilibrium is locally asymptotically stable. 


\section{Appendix B: Proof of Theorem 4.2}

The Jacobian matrix of the system (2.1) at the susceptible extinction equilibrium is

$$
\mathbf{D}=\left(\begin{array}{cc}
\mathbf{D}_{1} & \mathbf{0} \\
\mathbf{D}_{3} & \mathbf{D}_{2}
\end{array}\right)
$$

where $\mathbf{D}_{1}, \mathbf{D}_{2}, \mathbf{D}_{3}, \mathbf{0}$ are $2 \times 2$ matrices and

$$
\begin{aligned}
& \mathbf{D}_{1}=\left(\begin{array}{cc}
-\left(\eta_{1}+\mu+m N_{2}\right) & \beta_{1} \\
\eta_{1} & -\left(v+\alpha+m N_{2}\right)
\end{array}\right), \\
& \mathbf{D}_{2}=\left(\begin{array}{cc}
-\left(\eta_{2}+\mu+\gamma+m\left(J_{2}^{*}+N_{2}\right)\right) & \beta_{2}-m J_{2}^{*} \\
\eta_{2}-m A_{2}^{*} & -\left(\alpha+\gamma+m\left(A_{2}^{*}+N_{2}\right)\right)
\end{array}\right), \\
& \mathbf{D}_{3}=\left(\begin{array}{cc}
-m J_{2}^{*} & -m J_{2} \\
-m A_{2}^{*} & v-m A_{2}^{*}
\end{array}\right) .
\end{aligned}
$$

Clearly, $\operatorname{tr}\left(\mathbf{D}_{1}\right)<0$ and

$$
\begin{aligned}
\operatorname{det}\left(\mathbf{D}_{1}\right) & =\left[\left(\eta_{1}+\mu\right) \alpha-\beta_{1} \eta_{1}\right]+\left(\eta_{1}+\mu\right)\left(v+m N_{2}\right)+m N_{2}\left(v+\alpha+m N_{2}\right) \\
& =\alpha\left(\eta_{1}+\mu\right)\left(1-R_{1}\right)+\left(\eta_{1}+\mu\right)\left(v+m N_{2}\right)+m N_{2}\left(v+\alpha+m N_{2}\right)>0,
\end{aligned}
$$

since $R_{1} \geq 1$. In consequence, $\mathbf{D}_{1}$ has negative eigenvalues.

For $\mathbf{D}_{2}$, we have $\operatorname{tr}\left(\mathbf{D}_{2}\right)<0$. The last two equations of system (2.1) imply

$$
\left(\alpha+\gamma+m A_{2}^{*}\right)\left(\eta_{2}+\mu+\gamma+m J_{2}^{*}\right)=\left(\eta_{2}-m A_{2}^{*}\right)\left(\beta_{2}-m J_{2}^{*}\right) .
$$

Hence

$$
\begin{aligned}
\operatorname{det}\left(\mathbf{D}_{2}\right)= & \left(\alpha+\gamma+m A_{2}^{*}\right)\left(\eta_{2}+\mu+\gamma+m J_{2}^{*}\right)+m N_{2}\left(\alpha+\gamma+m\left(A_{2}^{*}+N_{2}\right)\right) \\
& +m N_{2}\left(\eta_{2}+\mu+\gamma+m J_{2}^{*}\right)-\left(\eta_{2}-m A_{2}^{*}\right)\left(\beta_{2}-m J_{2}^{*}\right) \\
= & m N_{2}\left(\eta_{2}+\mu+2 \gamma+\alpha+2 m N_{2}\right)>0 .
\end{aligned}
$$

Hence $\mathbf{D}_{2}$ has negative eigenvalues. Therefore, the susceptible extinction equilibrium is locally asymptotically stable.

\section{Appendix C: Proof of Theorem 4.3}

We show now that if $R_{1}>\left(\eta_{1}+\mu\right)(v+\alpha) /\left(\eta_{1} \alpha\right)$, then the trivial solution of the given system is a repeller in the sense that $\lim _{\sup _{t \rightarrow \infty}} N(t)>0$. We will prove this by contradiction. Assume that $\lim _{\sup _{t \rightarrow \infty}} N(t)=0$. Let $x=A_{1}+r J_{1}$, with $r \in(0,1)$ to be chosen later. The derivative of $x$ along a solution of the system $(2.1)$ is

$$
\begin{aligned}
x^{\prime} & \geq\left(\frac{\eta_{1}}{r}-\left(\eta_{1}+\mu\right)\right) r J_{1}+\left(r \frac{\beta_{1} \eta_{1}}{\eta_{1}+\mu}-v-\alpha\right) A_{1}-m x N \\
& =\left(\frac{\eta_{1}}{r}-\left(\eta_{1}+\mu\right)\right) r J_{1}+\left(r \alpha R_{1}-v-\alpha\right) A_{1}-m x N
\end{aligned}
$$

Since $R_{1}>\left(\eta_{1}+\mu\right)(v+\alpha) /\left(\eta_{1} \alpha\right)$, there exists an $0<\epsilon<1$ such that

$$
\eta_{1} \alpha R_{1}>\left(\eta_{1}+\mu+\epsilon\right)(v+\alpha+\epsilon) .
$$


Then, we can choose $r$ such that

$$
\frac{v+\alpha+\epsilon}{\alpha R_{1}}<r<\frac{\eta_{1}}{\eta_{1}+\mu+\epsilon}<1 .
$$

We thus have

$$
x^{\prime}>\epsilon x-m x N=x(\epsilon-m N) .
$$

If $\lim \sup _{t \rightarrow \infty} N(t)=0$, then $x \rightarrow \infty$, but this is a contradiction since $x=A_{1}+r J_{1}<$ $N$. Therefore, $\lim \sup _{t \rightarrow \infty} N(t)>0$.

Consider the following potential Liapunov function

$$
V(X)=\varrho_{2} J_{2}+A_{2}, \quad X=\left(J_{1}, A_{1}, J_{2}, A_{2}\right) \in \mathbb{R}_{+}^{4},
$$

with $\varrho_{2}=\eta_{2} /\left(\eta_{2}+\mu+\gamma\right)$. Then

$$
\dot{V}(X) \leq(\alpha+\gamma-v)\left(\frac{\xi \beta_{2} \eta_{2}}{\left(\eta_{2}+\mu+\gamma\right)(\alpha+\gamma-v)}-1\right) A_{2}-m V N
$$

Notice that he hypothesis $R_{2} \leq 1-R_{0}$ is equivalent to $\frac{\xi \beta_{2} \eta_{2}}{\left(\eta_{2}+\mu+\gamma\right)(\alpha+\gamma-v)}<1$. Hence $\dot{V}(X) \leq 0$ for $X \in \mathbb{R}_{+}^{4}$. This shows that $V$ is a Liapunov function with our selection of $\varrho_{2}$. We will now apply the Lyapunov-LaSalle's invariance principle. Clearly,

$$
E=\left\{X \in \mathbb{R}_{+}^{4}: \dot{V}(X)=0\right\}=\left\{\left(J_{1}, A_{1}, 0,0\right): J_{1} \geq 0, A_{1} \geq 0\right\}
$$

is an invariant set. As a consequence, the largest invariant set $M$ in $E$ is $E$. Hence all positive solutions of system (2.1) tend to $E$.

In the following, we will apply the Bendixson-Dulac criterion to show that positive solutions of the limiting system

$$
\left\{\begin{array}{l}
J_{1}^{\prime}=\beta_{1} A_{1}-\left(\eta_{1}+\mu\right) J_{1}-m J_{1}\left(J_{1}+A_{1}\right) \equiv F\left(J_{1}, A_{1}\right), \\
A_{1}^{\prime}=\eta_{1} J_{1}-\alpha A_{1}-m A_{1}\left(J_{1}+A_{1}\right) \equiv G\left(J_{1}, A_{1}\right),
\end{array}\right.
$$

will tend to the positive equilibrium $E_{1}=\left(J_{1}^{*}, A_{1}^{*}\right)$. An application of the limiting equation theory ${ }^{9}$ allows us to conclude that positive solutions of the system (2.1) tend to the disease-free equilibrium.

Let $\rho\left(J_{1}, A_{1}\right)=\frac{1}{J_{1} A_{1}}$. We have $\frac{\partial(\rho F)}{\partial J_{1}}+\frac{\partial(\rho G)}{\partial A_{1}}<0$ for all $\left(J_{1}, A_{1}\right) \in(0, \infty)^{2}$. By the Bendixson-Dulac criterium, we see that there are no non-trivial periodic orbits in $(0, \infty)^{2}$. The solutions of system (C.1) are bounded and there are only two equilibrium points, the trivial one and the positive one. Recall that the trivial equilibrium is a repeller for system (2.1) therefore the trivial equilibrium of the system (C.1) is also a repeller. In addition the proof of Theorem 4.1 shows that the positive steady state of the system (C.1) is locally asymptotically stable. These statement together show that the $\omega$-limit set of a bounded positive solution of system (C.1) is the only positive equilibrium $\left(J_{1}^{*}, A_{1}^{*}\right)$. Therefore the disease-free equilibrium $\left(J_{1}^{*}, A_{1}^{*}, 0,0\right)$ is a global attractor of the system $(2.1)$. 


\section{Appendix D: Proof of Theorem 4.4}

Consider $y=A_{2}+s J_{2}$, with $s \in(0,1)$ to be chosen later. The derivative of $y$ along a solution of the system $(2.1)$ is

$$
\begin{aligned}
y^{\prime} & \geq\left(\frac{\eta_{2}}{s}-\left(\eta_{2}+\mu+\gamma\right)\right) s J_{2}+\left(\frac{\beta_{2} \eta_{2}}{\eta_{2}+\mu+\gamma} s-(\alpha+\gamma)\right) A_{2}-m y N \\
& =\left(\frac{\eta_{2}}{s}-\left(\eta_{2}+\mu+\gamma\right)\right) s J_{2}+(\alpha+\gamma)\left(s R_{0}-1\right) A_{2}-m y N
\end{aligned}
$$

Since $R_{0}>\frac{\eta_{2}+\mu+\gamma}{\eta_{2}}=\frac{\left(\eta_{2}+\mu+\gamma\right)(\alpha+\gamma)}{\eta_{2}(\alpha+\gamma)}$, there exists an $0<\tilde{\epsilon}<1$ such that

$$
\eta_{2}(\alpha+\gamma) R_{2}>\left(\eta_{2}+\mu+\gamma+\tilde{\epsilon}\right)(\alpha+\gamma+\tilde{\epsilon}) .
$$

We choose $s$ such that

$$
\frac{\alpha+\gamma+\tilde{\epsilon}(\alpha+\gamma) R_{2}}{<} s<\frac{\eta_{2}}{\eta_{2}+\mu+\gamma+\tilde{\epsilon}}<1 .
$$

Then $y^{\prime}>\tilde{\epsilon} y-m y N=y(\tilde{\epsilon}-m N)$. If $\lim \sup N(t)=0$ then $y \rightarrow \infty$, but this is a contradiction since $y=A_{2}+s J_{2}<N$ is bounded. Hence, $\limsup _{t \rightarrow \infty} N(t)>0$.

Consider the following potential Liapunov function

$$
V(X)=\varrho_{1} J_{1}+A_{1}, \quad X=\left(J_{1}, A_{1}, J_{2}, A_{2}\right),
$$

with $\varrho_{1}=\eta_{1} /\left(\eta_{1}+\mu\right)$. The derivative of $V$ along a solution of the system $(2.1)$ is

$$
\dot{V}(X)=\alpha\left(R_{1}-1\right) A_{1}-\frac{\nu A_{1} A_{2}}{A}-m V N
$$

Since $R_{1} \leq 1$, we see that $\dot{V}(X) \leq 0$ for $X \in \mathbb{R}_{+}^{4}$. An application of the LiapunovLaSalle's invariance principle shows that positive solutions tend to

$$
E=\left\{X \in \mathbb{R}_{+}^{4}: \dot{V}(X)=0\right\}=\left\{\left(0,0, J_{2}, A_{2}\right): J_{2} \geq 0, A_{2} \geq 0\right\}
$$

as $t \rightarrow \infty$. Likewise, through the standard application of Bendixson-Dulac criterion and phase plane analysis, we can show that positive solutions of the limiting $J_{2} A_{2}$-system tend to the positive equilibrium $\left(J_{2}^{*}, A_{2}^{*}\right)$. Therefore, the susceptible extinction equilibrium $\left(0,0, J_{2}^{*}, A_{2}^{*}\right)$ is a global attractor of the system (2.1).

\section{Appendix E: Proof of Theorem 4.5}

Consider the following Liapunov function in $\mathbb{R}_{+}^{4}$,

$$
V(X)=\varrho_{1} J_{1}+A_{1}+\varrho_{2} J_{2}+A_{2}, \quad X=\left(J_{1}, A_{1}, J_{2}, A_{2}\right),
$$


with $\varrho_{1}=\frac{\eta_{1}}{\eta_{1}+\mu}<1$ and $\varrho_{2}=\frac{\eta_{2}}{\eta_{2}+\mu+\gamma}<1$. The derivative of $V$ along a solution of the system $(2.1)$ is

$$
\dot{V}(X)=\alpha\left(R_{1}-1\right) A_{1}+(\alpha+\gamma)\left(R_{0}-1\right) A_{2}-m V N \leq 0
$$

for $X \in \mathbb{R}_{+}^{4}$. The zero set for $\dot{V}(X)$ is

$$
E=\left\{X \in \mathbb{R}_{+}^{4}: \dot{V}(X)=0\right\}=\{(0,0,0,0)\}
$$

which is an invariant set. By the LaSalle Liapunov theorem, we conclude that all positive solutions of system (2.1) tend to $E$ as $t \rightarrow \infty$. 